\title{
Características clínicas y comorbilidades de pacientes con lupus eritematoso sistémico en niños y adultos
}

\author{
Coronado-Alvarado CD ${ }^{1}$, Gámez-Saiz IL ${ }^{1}$, Sotelo-Cruz N²
}

\begin{abstract}
Resumen
INTRODUCCIÓN: el lupus eritematoso sistémico es una enfermedad autoinmune crónica; se presenta mayormente en mujeres en etapa reproductiva y es menos frecuente en niños.
\end{abstract}

OBJETIVO: comparar las manifestaciones clínicas, comorbilidades y farmacoterapia en niños y adultos con lupus eritematoso sistémico (LES).

MATERIAL Y MÉTODOS: se seleccionaron 127 expedientes clínicos de pacientes con diagnóstico de LES; 36 menores de edad de un hospital pediátrico (grupo A) y 91 adultos de un hospital general (grupo B). Recolectamos información respecto a las manifestaciones clínicas al diagnóstico en los niños y adultos, así como las comorbilidades relacionadas y mortalidad, atendiendo los criterios de la American College of Rheumatology, también del tipo de medicamentos empleados. Estadísticas utilizadas: cálculo de frecuencias, porcentajes, la prueba de ji cuadrada, prueba exacta de Fisher, razón de momios e intervalos de confianza.

RESULTADOS: en niños predominaron: eritema malar $(p=<0.01)$, vasculitis $(p<0.01)$, fotosensibilidad $(p<0.01)$, síndrome nefrítico $(p<0.01)$, alteraciones leucocitarias $(p<0.01)$, derrame pleural, anemia, derrame pericárdico, sepsis y hepatopatía; además, el número de medicamentos empleados fue mayor. En adultos predominaron: artralgia $(p<0.01)$, pancitopenia $(p=0.025)$, alopecia $(p<0.01)$, sinovitis $(p=0.044)$ y falla renal crónica $(p=0.04)$. Los fármacos usados en el grupo A fueron: hidroxicloroquina $(p<0.01)$, glucocorticoides $(p=0.14)$ y micofenolato $(p=0.28)$, la mortalidad en niños fue de $25 \%$ y en 91 adultos ocurrió en $5(0.05 \%)$.

CONCLUSIONES: las manifestaciones clínicas en niños muestran mayor lesión cutánea inicial y vasculitis, daño renal, cardiovascular, pulmonar e infecciones; en los adultos predominan afecciones articulares y falla renal crónica. Los niños reciben mayor número de fármacos y su mortalidad es mayor.

PALABRAS CLAVE: lupus eritematoso sistémico, manifestaciones clínicas, niños, adultos.
${ }^{1}$ Alumno de la Lic. en Medicina, $9^{\circ}$ semestre. 2Profesor Investigador Titular "B".

Departamento de Medicina Universidad de Sonora.

Recibido: 20 de octubre del 2016

Aceptado: 4 de abril del 2017

\section{Correspondencia}

Norberto Sotelo-Cruz

nsotelo51@gmail.com

Este artículo debe citarse como

Coronado-Alvarado CD, Gámez-Saiz IL, SoteloCruz N. Características clínicas y comorbilidades de pacientes con lupus eritematoso sistémico en niños y adultos. Acta Pediatr Mex. 2018;39(1):1-12. 


\section{Clinical features and comorbidities of systemic lupus, differences between children and adults.}

\author{
Coronado-Alvarado CD ${ }^{1}$, Gámez-Saiz IL ${ }^{1}$, Sotelo-Cruz N²
}

\begin{abstract}
INTRODUCTION: Systemic lupus erythematosus, is a chronic autoimmune disease that occurs mostly in women in the reproductive stage, is less common in children.

OBJECTIVE: To compare the clinical features, comorbidities and pharmacotherapy of the patients diagnosed with systemic lupus erythematous (SLE).
\end{abstract}

MATERIALS AND METHODS: We reviewed 127 clinical records of patients diagnosed with SLE, 36 patients were from a paediatric hospital (group A) and 91 from a general hospital (group B). The variables explored included the clinical manifestations in children at diagnosis and those of adults as well as the comorbidities related to the disease and mortality, according criteria from American College of Rheumatology, also the type of medication used. The statistical procedures were frequencies and percentages, chi-squared test, Fisher's exact test, odds ratios and confidence intervals.

RESULTS: The predominant findings in children were; malar rash $(p=<0.01)$, vasculitis $(p<0.01)$, photosensitivity $(p<0.01)$, nephritic syndrome $(p<0.01)$, leukocyte's abnormalities $(p<0.01)$, pleural effusion, anaemia, thrombocytopenia, pericardial effusion, sepsis and liver disease; beside, the number of medication used was higher. In adults the findings were: arthralgia $(p<0.01)$, pancytopenia $(p=0.025)$, alopecia $(p=0.04)$, synovitis and chronic kidney disease $(p=0.04)$. The drugs used in-group A were hydroxychloroquine $(p<0.01)$, glucocorticoids $(p=0.14)$ and mycophenolate $(p=0.28)$, the mortality in children was $25 \%$ and in 91 adults were 5 patients $(0.05 \%)$.

CONCLUSIONS: In children, clinical manifestations show a greater initial, skin and vasculitis, renal damage, cardiovascular, pulmonary, and infection condition than in adults, in these predominate articular affections and chronic renal failure. Children received more medication; the mortality is higher than adults are.

KEYWORDS: systemic lupus erythematous; clinical manifestations; children; adults
${ }^{1}$ Alumno de la Lic. en Medicina, $9^{\circ}$ semestre.

2Profesor Investigador Titular "B".

Departamento de Medicina Universidad de Sonora.

Correspondence

Norberto Sotelo-Cruz

nsotelo51@gmail.com 


\section{INTRODUCCIÓN}

El lupus eritematoso sistémico es una enfermedad autoinmune, crónica, inflamatoria, que tiene manifestaciones clínicas muy diversas que afectan la función de distintos órganos y sistemas del cuerpo. ${ }^{1}$

En México se ha reportado una prevalencia de $0.06 \%$ para esta enfermedad; la incidencia se ha estimado entre 1.8 y 7.6 casos por cada $100,000 .^{2}$ La supervivencia a los 5 años postdiagnóstico ha mejorado de forma progresiva, probablemente debido a una mejor comprensión de la enfermedad, a la aparición de nuevas terapias inmunosupresoras y de mejores tratamientos para el control de los daños.

Se tiene el antecedente de que en 1950, con la administración de esteroides, se redujo la mortalidad del LES, lo que permitió prolongar la vida de los pacientes y realizar estudios, así como ajustes en los esquemas terapéuticos que han continuado impactando favorablemente las tasas de mortalidad; la supervivencia se ha incrementado en las dos últimas décadas de 10 hasta 15 años, superior al 85\%, y de 65\% a 20 años.

Con el paso de los años se ha modificado el curso natural de la enfermedad propiciando una transición en las complicaciones y manifestaciones clínicas de pacientes con LES a los padecimientos crónicos, como consecuencia del aumento en la esperanza de vida. En los niños, la mortalidad por LES está asociada con infecciones, mientras que en etapas tardías de la vida se relaciona enfermedad cardiovascular. ${ }^{1,3-6}$

Se ha reportado que hasta $20 \%$ de los pacientes con LES inician su enfermedad durante la edad pediátrica, y que en éstos la actividad de la enfermedad es mayor, pues presentan más rápido manifestaciones clínicas y complicaciones que ponen en peligro su vida. ${ }^{3-6}$
Debido a que en la literatura se reportan variaciones en las manifestaciones clínicas del LES, entre niños y adultos, se consideró prudente analizar lo que ocurre en nuestro ámbito de trabajo; además de que existe escasa información de esta naturaleza en el país y primordialmente en esta región.

\section{OBJETIVO}

Comparar las características clínicas y comorbilidades del LES en pacientes de edades pediátricas y adultos, tomando para ello como referencia dos centros de atención médica de concentración de la Secretaria de Salud en el estado de Sonora, México.

\section{MATERIAL Y MÉTODOS}

\section{Diseño del estudio}

Estudio retrospectivo, observacional, descriptivo y analítico de casos clínicos de pacientes con LES atendidos en los servicios de medicina interna y reumatología de dos instituciones: una para atención pediátrica y un hospital general.

\section{Selección de la muestra y variables estudiadas}

Se obtuvo un listado con los pacientes diagnosticados con LES en los últimos 10 años en ambas instituciones, se seleccionaron 36 expedientes del hospital pediátrico de forma aleatoria mediante el programa Excel. Se eligieron a 100 de 243 pacientes adultos atendidos por LES, de los cuales nueve fueron descartados por no tener información completa. 127 expedientes se clasificaron según el grupo etario al que atendía cada hospital. Se definieron como grupo A al integrado por niños con 36 pacientes atendidos en el hospital pediátrico y al segundo grupo B, conformado por 91 adultos del hospital general.

A partir de los expedientes de los pacientes se analizaron las manifestaciones clínicas que 
presentaban al momento de su diagnóstico, las comorbilidades fueron agrupadas en distintas categorías según el tipo de órgano o sistema al que afectaban; se siguieron los criterios del American College of Rheumatology. También obtuvimos información sobre el tratamiento inmunosupresor y presencia del síndrome de polifarmacia, considerando el uso concomitante de tres o más fármacos en dichos pacientes; adicionalmente se registró el número total de pacientes que fallecieron y las causas, cuyas características y factores relacionados serán motivo de otro informe. No se analizaron aspectos como índices de actividad lúpica ni comparaciones entre respuestas a fármacos, comorbilidad y supervivencia.

\section{Análisis estadístico}

La información recabada fue analizada por medio del paquete estadístico SPSS 20. Se hizo el cálculo de frecuencias y porcentajes, así como pruebas de hipótesis para encontrar asociaciones significativas entre el grupo etario de los pacientes y las entidades clínicas reportadas empleando la prueba de $\chi^{2} y$, en caso de ser necesaria, cuando en alguna de las categorías el número de pacientes fuera inferior a cinco, se utilizó la prueba exacta de Fisher. Además, para las principales manifestaciones clínicas que se asociaron con la mortalidad de los pacientes con $\mathrm{LES}^{6-13}$ se obtuvieron las razones de momios (RM) y sus respectivos intervalos de confianza al 95\% para conocer el riesgo en los niños para padecer comorbilidades, comparados con el grupo de adultos.

El estudio fue autorizado por los departamentos de enseñanza e investigación, considerándolo sin riesgos y aprobado por los comités internos de ética de ambas instituciones.

\section{RESULTADOS}

De los 127 expedientes 36 pertenecían al grupo $\mathrm{A}$; en éste, 26 (72.2\%) correspondían a pacientes femeninos, para una proporción mujer:hombre de 2.6:1. El paciente pediátrico de menor edad tenía 6 años cumplidos al momento del estudio; mientras que el mayor contaba con 19 años. La edad media para los niños fue de 13.6 con una desviación estándar de \pm 3 3.3. El grupo B estuvo compuesto por 91 sujetos y de éstos 87 (95.6\%) eran mujeres, la proporción mujer:hombre fue 21.7:1; en este mismo grupo el paciente de menor edad tenía 17 años cumplidos, mientras que el mayor era de 91 años. La edad media para los adultos fue de 39.5 años con una desviación estándar de \pm 13.5 .

\section{Manifestaciones clínicas observadas}

Los niños presentaron más afecciones cutáneas, hematológicas, cardiovasculares e infecciones; en los adultos hubo mayor frecuencia de enfermedad reumatológica (Cuadro 1). En lo que respecta a las manifestaciones clínicas que se registraron en ambos grupos sí se encontraron algunas diferencias significativas (Cuadro 2). Las afecciones cutáneas fueron más evidentes en los niños: fotosensibilidad $(p<0.01)$ y eritema malar $(p=<0.01)$ pero no para el caso de eritema discoide, éste solo estuvo presente en $5.5 \%$ de los niños $(p=0.11)$ mientras que $14.3 \%$ de los adultos lo presentó.

Las alteraciones articulares predominaron más en los adultos que en los niños encabezadas por las artralgias $(p<0.01)$ y la sinovitis $(p=0.044)$. No hubo diferencias por grupo etario en cuanto a la artritis $(p=0.062)$ ni a la artrosis $(p=0.71)$.

Para las nefropatías el síndrome nefrítico fue predominante en los niños $(p<0.01)$ y la falla renal crónica en los adultos $(p=0.04)$. No se encontraron diferencias en cuanto al síndrome nefrótico $(p=0.25)$ ni en la falla renal aguda $(p=0.51)$.

Respecto de las alteraciones hematológicas en los niños fueron más frecuentes la anemia $(p=<0.04)$, las alteraciones leucocitarias 
Cuadro 1. Hallazgos clínicos en niños y adultos, con diagnóstico de lupus eritematoso sistémico, atendidos en dos hospitales* de Sonora (2016)

\begin{tabular}{|c|c|c|c|c|c|}
\hline \multirow{2}{*}{ Variable estudiada } & \multicolumn{2}{|c|}{$\begin{array}{c}\text { Grupo A } \\
(n=36)\end{array}$} & \multicolumn{2}{|c|}{$\begin{array}{c}\text { Grupo B } \\
(n=91)\end{array}$} & \multirow[b]{2}{*}{$\overline{p^{* *}}$} \\
\hline & $\mathbf{n}$ & $\%$ & $\mathbf{n}$ & $\%$ & \\
\hline \multicolumn{6}{|l|}{ Afecciones articulares } \\
\hline $\begin{array}{l}\text { Sí } \\
\text { No }\end{array}$ & $\begin{array}{l}24 \\
12\end{array}$ & $\begin{array}{l}66.7 \\
33.3\end{array}$ & $\begin{array}{l}54 \\
37\end{array}$ & $\begin{array}{l}59.3 \\
40.7\end{array}$ & 0.280 \\
\hline \multicolumn{6}{|l|}{ Afecciones cutáneas } \\
\hline $\begin{array}{l}\text { Sí } \\
\text { No }\end{array}$ & $\begin{array}{l}26 \\
10\end{array}$ & $\begin{array}{l}72.2 \\
27.8\end{array}$ & $\begin{array}{l}36 \\
55\end{array}$ & $\begin{array}{l}39.6 \\
60.4\end{array}$ & $<0.01$ \\
\hline \multicolumn{6}{|l|}{ Afecciones renales } \\
\hline $\begin{array}{l}\text { Sí } \\
\text { No }\end{array}$ & $\begin{array}{l}16 \\
20\end{array}$ & $\begin{array}{l}44.4 \\
55.6\end{array}$ & $\begin{array}{l}31 \\
60\end{array}$ & $\begin{array}{l}34.1 \\
65.9\end{array}$ & 0.187 \\
\hline \multicolumn{6}{|c|}{ Afecciones hemáticas } \\
\hline $\begin{array}{l}\text { Sí } \\
\text { No }\end{array}$ & $\begin{array}{c}35 \\
1\end{array}$ & $\begin{array}{c}97.2 \\
2.8\end{array}$ & $\begin{array}{l}66 \\
25\end{array}$ & $\begin{array}{l}72.5 \\
27.5\end{array}$ & $<0.01$ \\
\hline \multicolumn{6}{|l|}{ Úlceras } \\
\hline $\begin{array}{l}\text { Sí } \\
\text { No }\end{array}$ & $\begin{array}{c}7 \\
29\end{array}$ & $\begin{array}{l}19.4 \\
80.6\end{array}$ & $\begin{array}{l}16 \\
75\end{array}$ & $\begin{array}{l}17.6 \\
82.4\end{array}$ & 0.494 \\
\hline \multicolumn{6}{|l|}{ Serositis } \\
\hline $\begin{array}{l}\text { Sí } \\
\text { No }\end{array}$ & $\begin{array}{l}13 \\
23\end{array}$ & $\begin{array}{l}36.1 \\
63.9\end{array}$ & $\begin{array}{l}14 \\
77\end{array}$ & $\begin{array}{l}15.4 \\
84.6\end{array}$ & 0.012 \\
\hline \multicolumn{6}{|l|}{ Infecciones } \\
\hline $\begin{array}{l}\text { Sí } \\
\text { No }\end{array}$ & $\begin{array}{l}21 \\
15\end{array}$ & $\begin{array}{l}58.3 \\
41.7\end{array}$ & $\begin{array}{l}26 \\
65\end{array}$ & $\begin{array}{l}28.6 \\
71.4\end{array}$ & $<0.01$ \\
\hline \multicolumn{6}{|c|}{ Afecciones neuropsiquiátricas } \\
\hline $\begin{array}{l}\text { Sí } \\
\text { No }\end{array}$ & $\begin{array}{c}7 \\
29\end{array}$ & $\begin{array}{l}19.4 \\
80.6\end{array}$ & $\begin{array}{l}26 \\
65\end{array}$ & $\begin{array}{l}28.6 \\
71.4\end{array}$ & 0.204 \\
\hline \multicolumn{6}{|c|}{ Comorbilidades reumatológicas (artritisreumatoide) } \\
\hline $\begin{array}{l}\text { Sí } \\
\text { No }\end{array}$ & $\begin{array}{c}5 \\
31\end{array}$ & $\begin{array}{l}13.9 \\
86.1\end{array}$ & $\begin{array}{l}37 \\
54\end{array}$ & $\begin{array}{l}40.7 \\
59.3\end{array}$ & $<0.01$ \\
\hline \multicolumn{6}{|c|}{ Afecciones gastrointestinales } \\
\hline $\begin{array}{l}\text { Sí } \\
\text { No }\end{array}$ & $\begin{array}{c}3 \\
33\end{array}$ & $\begin{array}{c}8.3 \\
91.7\end{array}$ & $\begin{array}{l}19 \\
72\end{array}$ & $\begin{array}{l}20.9 \\
79.1\end{array}$ & 0.072 \\
\hline \multicolumn{6}{|c|}{ Afecciones oftalmológicas } \\
\hline $\begin{array}{l}\text { Sí } \\
\text { No }\end{array}$ & $\begin{array}{c}0 \\
36\end{array}$ & $\begin{array}{c}0 \\
100\end{array}$ & $\begin{array}{c}6 \\
85\end{array}$ & $\begin{array}{c}6.6 \\
93.4\end{array}$ & 0.129 \\
\hline \multicolumn{6}{|c|}{ Afecciones cardiovasculares } \\
\hline $\begin{array}{l}\text { Sí } \\
\text { No }\end{array}$ & $\begin{array}{l}19 \\
17\end{array}$ & $\begin{array}{l}52.8 \\
47.2\end{array}$ & $\begin{array}{l}23 \\
68\end{array}$ & $\begin{array}{l}25.3 \\
74.7\end{array}$ & $<0.01$ \\
\hline
\end{tabular}

*Hospital Infantil del Estado de Sonora (HIES), Hospital General del Estado de Sonora (HGES).

**El valor de $p$ se obtuvo con la prueba de ji cuadrada, excepto en los casos con celdas con valores menores a 5, donde se empleó la prueba exacta de Fisher. (leucopenia) $(p<0.01)$ y la trombocitopenia $(p=0.01)$; los adultos tuvieron mayor número de casos con bicitopenia $(p=<0.32$ ) y opancitopenia $(p=0.02)$, sin observar un mayor número de casos de púrpura en los adultos $(p=0.68)$.

Los niños tuvieron serositis con mayor frecuencia que los adultos: derrame pleural $(p<0.01)$, derrame pericárdico $(p<0.01)$, ascitis $(p \leq 0.63)$ y anasarca $(p \leq 0.28)$. En ningún adulto se reportó anasarca o derrame pericárdico.

En los niños los episodios de sepsis fueron: $(p=0.03)$ y neumonía $(p \leq 0.27)$; mientras que en los adultos de herpes $(p=0.25)$, infecciones de vías urinarias $(p=0.09)$ y candidiasis $(p=0.36)$.

Ninguna de las afecciones neuropsiquiátricas reportadas tuvieron un valor estadísticamente significativo entre niños o adultos, a pesar de que se observó que los niños presentaban más convulsiones $(11.1 \%$ en niños contra $2.2 \%$ en adultos); trastorno de ansiedad $2.8 \%$ contra $1.1 \%$ y psicosis $8.3 \%$ contra $3.3 \%$. Por otro lado, los adultos presentaron algún tipo de apoplejía, $2.2 \%$, contra $0 \%$ en niños; el trastorno bipolar fue $2.2 \%$ contra $0 \%$ y trastorno de depresión mayor $12.1 \%$ contra $2.8 \%$, respectivamente.

La alopecia predominó en los adultos $(p<0.01)$. No se reportaron casos de niños con mialgias, síndrome de Sjögren, dermatopolimiositis, artritis reumatoide, esclerosis nodular, síndrome de CREST, ni hipotiroidismo, y tuvieron una menor frecuencia de manifestaciones del fenómeno de Raynaud, al compararlos con los adultos $(8.3 \%$ en niños contra $15.4 \%$ en adultos) $p=0.22$. Se encontró una mayor frecuencia en niños de síndrome antifosfolípidos (2.8\% contra 1.1\%, $p=0.48)$.

De las comorbilidades del aparato digestivo se encontró una diferencia significativa en daño hepático en el grupo de niños $(p<0.02)$.

En los adultos se reportaron ocho pacientes con gastritis, siete pacientes con sangrado de tubo 
Cuadro 2. Hallazgos clínicos significativos según grupo etario, en pacientes con lupus eritematoso sistémico, atendidos en dos hospitales* de Sonora (2016). Continúa en la siguiente columna

\begin{tabular}{|c|c|c|c|c|c|}
\hline \multirow{2}{*}{ Variable estudiada } & \multicolumn{2}{|c|}{$\begin{array}{c}\text { Grupo A } \\
(n=36)\end{array}$} & \multicolumn{2}{|c|}{$\begin{array}{c}\text { Grupo B } \\
(n=91)\end{array}$} & \multirow[b]{2}{*}{$p^{* *}$} \\
\hline & $\mathbf{n}$ & $\%$ & $\mathbf{n}$ & $\%$ & \\
\hline \multicolumn{6}{|l|}{ Sinovitis } \\
\hline $\begin{array}{l}\text { Sí } \\
\text { No }\end{array}$ & $\begin{array}{c}0 \\
36\end{array}$ & $\begin{array}{c}0 \\
100\end{array}$ & $\begin{array}{c}9 \\
82\end{array}$ & $\begin{array}{c}9.9 \\
90.1\end{array}$ & 0.044 \\
\hline \multicolumn{6}{|l|}{ Artralgia } \\
\hline $\begin{array}{l}\text { Sí } \\
\text { No }\end{array}$ & $\begin{array}{l}19 \\
17\end{array}$ & $\begin{array}{l}52.8 \\
47.2\end{array}$ & $\begin{array}{l}23 \\
68\end{array}$ & $\begin{array}{l}74.7 \\
25.3\end{array}$ & $<0.01$ \\
\hline \multicolumn{6}{|l|}{ Fotosensibilidad } \\
\hline $\begin{array}{l}\text { Sí } \\
\text { No }\end{array}$ & $\begin{array}{l}16 \\
20\end{array}$ & $\begin{array}{l}44.4 \\
55.6\end{array}$ & $\begin{array}{l}15 \\
76\end{array}$ & $\begin{array}{l}16.5 \\
83.5\end{array}$ & $<0.01$ \\
\hline \multicolumn{6}{|l|}{ Eritema malar } \\
\hline $\begin{array}{l}\text { Sí } \\
\text { No }\end{array}$ & $\begin{array}{l}21 \\
15\end{array}$ & $\begin{array}{l}58.3 \\
41.7\end{array}$ & $\begin{array}{l}19 \\
72\end{array}$ & $\begin{array}{c}20.79 \\
79.1\end{array}$ & $<0.01$ \\
\hline \multicolumn{6}{|l|}{ Síndrome nefrítico } \\
\hline $\begin{array}{l}\text { Sí } \\
\text { No }\end{array}$ & $\begin{array}{l}16 \\
20\end{array}$ & $\begin{array}{l}44.4 \\
55.6\end{array}$ & $\begin{array}{l}16 \\
75\end{array}$ & $\begin{array}{l}17.6 \\
82.4\end{array}$ & $<0.01$ \\
\hline \multicolumn{6}{|l|}{ Falla renal crónica } \\
\hline $\begin{array}{l}\text { Sí } \\
\text { No }\end{array}$ & $\begin{array}{c}0 \\
36\end{array}$ & $\begin{array}{c}0 \\
100\end{array}$ & $\begin{array}{c}9 \\
82\end{array}$ & $\begin{array}{c}9.9 \\
90.1\end{array}$ & 0.044 \\
\hline \multicolumn{6}{|c|}{ Alteraciones leucocitarias } \\
\hline $\begin{array}{l}\text { Sí } \\
\text { No }\end{array}$ & $\begin{array}{l}16 \\
20\end{array}$ & $\begin{array}{l}44.4 \\
55.6\end{array}$ & $\begin{array}{c}7 \\
84\end{array}$ & $\begin{array}{c}7.7 \\
92.3\end{array}$ & $<0.01$ \\
\hline \multicolumn{6}{|l|}{ Trombocitopenia } \\
\hline $\begin{array}{l}\text { Sí } \\
\text { No }\end{array}$ & $\begin{array}{c}9 \\
27\end{array}$ & $\begin{array}{l}25 \\
75\end{array}$ & $\begin{array}{c}7 \\
84\end{array}$ & $\begin{array}{c}7.7 \\
92.3\end{array}$ & 0.012 \\
\hline \multicolumn{6}{|l|}{ Anemia } \\
\hline $\begin{array}{l}\text { Sí } \\
\text { No }\end{array}$ & $\begin{array}{l}11 \\
25\end{array}$ & $\begin{array}{l}30.6 \\
69.4\end{array}$ & $\begin{array}{l}14 \\
77\end{array}$ & $\begin{array}{l}15.4 \\
84.6\end{array}$ & 0.048 \\
\hline \multicolumn{6}{|l|}{ Pancitopenia } \\
\hline $\begin{array}{l}\text { Sí } \\
\text { No }\end{array}$ & $\begin{array}{c}3 \\
33\end{array}$ & $\begin{array}{c}8.3 \\
91.7\end{array}$ & $\begin{array}{l}23 \\
68\end{array}$ & $\begin{array}{l}25.3 \\
74.7\end{array}$ & 0.025 \\
\hline \multicolumn{6}{|l|}{ Derrame pleural } \\
\hline $\begin{array}{l}\text { Sí } \\
\text { No }\end{array}$ & $\begin{array}{l}13 \\
23\end{array}$ & $\begin{array}{l}36.1 \\
63.9\end{array}$ & $\begin{array}{l}12 \\
79\end{array}$ & $\begin{array}{l}13.2 \\
86.8\end{array}$ & $<0.01$ \\
\hline \multicolumn{6}{|l|}{ Derrame pericárdico } \\
\hline $\begin{array}{l}\text { Sí } \\
\text { No }\end{array}$ & $\begin{array}{c}9 \\
27\end{array}$ & $\begin{array}{l}25 \\
75\end{array}$ & $\begin{array}{c}0 \\
91\end{array}$ & $\begin{array}{c}0 \\
100\end{array}$ & $<0.01$ \\
\hline \multicolumn{6}{|l|}{ Sepsis } \\
\hline $\begin{array}{l}\text { Sí } \\
\text { No }\end{array}$ & $\begin{array}{c}6 \\
30\end{array}$ & $\begin{array}{l}16.7 \\
83.3\end{array}$ & $\begin{array}{c}4 \\
87\end{array}$ & $\begin{array}{c}4.4 \\
95.6\end{array}$ & 0.03 \\
\hline
\end{tabular}

Cuadro 2. Hallazgos clínicos significativos según grupo etario, en pacientes con lupus eritematoso sistémico, atendidos en dos hospitales* de Sonora (2016). Continuación

\begin{tabular}{l|c|c|c|c|c}
\multirow{2}{*}{ Variable estudiada } & \multicolumn{2}{|c|}{$\begin{array}{c}\text { Grupo A } \\
(\mathbf{n = 3 6 )}\end{array}$} & \multicolumn{2}{c|}{$\begin{array}{c}\text { Grupo B } \\
(\mathbf{n = 9 1 )}\end{array}$} & \\
\cline { 2 - 6 } & $\mathbf{n}$ & $\%$ & $\mathbf{n}$ & $\%$ & $\overline{\boldsymbol{p}^{* *}}$ \\
\hline Alopecia & & & & & \\
$\quad$ Sí & 0 & 0 & 16 & 17.6 & $<0.01$ \\
$\quad$ No & 36 & 100 & 75 & 82.4 & \\
Hepatopatía & & & & & \\
$\quad$ Sí & 3 & 8.3 & 0 & 0 & 0.021 \\
$\quad$ No & 33 & 91.7 & 91 & 100 & \\
Vasculitis & & & & & \\
$\quad$ Sí & 19 & 52.8 & 12 & 13.2 & $<0.01$ \\
$\quad$ No & 17 & 47.2 & 79 & 86.8 & \\
\end{tabular}

*Hospital Infantil del Estado de Sonora (HIES), Hospital General del Estado de Sonora (HGES).

**El valor de $p$ se obtuvo con la prueba de ji cuadrada, excepto en los casos con celdas con valores menores a 5, donde se empleó la prueba exacta de Fisher.

digestivo, cuatro pacientes con enfermedad de reflujo gastroesofágico (estas comorbilidades pueden corresponder a efectos adversos del tratamiento) y en dos pacientes várices esofágicas a afección secundaria a daño hepático e hipertensión portal.

La vasculitis predominó en el grupo A con una proporción mayor que en adultos $(p<0.01)$. La hipertensión arterial sistémica entre niños $(8.3 \%)$ y adultos $(5.3 \%)$, no fue estadísticamente significativa $(p=0.4)$.

Entre las cardiopatías que sólo fueron reportadas en el grupo B se encuentran las siguientes: miocarditis, endocarditis, hipertensión arterial pulmonar, infarto agudo al miocardio, fibrilación auricular, insuficiencia venosa e insuficiencia cardíaca congestiva.

Se procuró estimar el riesgo de los niños a presentar determinadas manifestaciones clínicas a través de la razón de momios (OR) y se observó 
que aquellas asociadas con mal pronóstico son más frecuentes en niños y con mortalidad en pacientes con LES según otros estudios. ${ }^{6-13} \mathrm{Tu}$ vimos: síndrome nefrítico (OR = 3.75; IC 95\%: 1.6-8.7), alteraciones hematológicas, leucopenia $(\mathrm{OR}=9.6$; IC 95\%: 3.4-26.4), derrame pleural $(\mathrm{OR}=3.72$; IC 95\%: 1.49-9.26), vasculitis (OR = 7.35; IC 95\%: 3.01-17.96), sepsis $(\mathrm{OR}=4.35 ;$ IC 95\%: 1.14-16.47), infecciones $(\mathrm{OR}=3.5 ; \mathrm{IC} 95 \%: 1.56-7.81)$ y afecciones cardiovasculares $(\mathrm{OR}=3.3$; IC 95\%: 1.47-7.4

\section{(Cuadro 3).}

Cuadro 3. Principales manifestaciones clínicas del lupus eritematoso sistémico asociadas con mortalidad según otros estudios, acorde al grupo etario de pacientes con este diagnóstico atendidos en dos hospitales de Sonora (2016)* Continúa en la siguiente columna

\begin{tabular}{|c|c|c|c|c|c|}
\hline \multirow{2}{*}{ Variable estudiada } & \multicolumn{2}{|c|}{$\begin{array}{c}\text { Grupo A } \\
(n=36)\end{array}$} & \multicolumn{2}{|c|}{$\begin{array}{c}\text { Grupo B } \\
(n=91)\end{array}$} & \multirow[b]{2}{*}{$\begin{array}{c}p^{* *}, \\
\text { OR IC } 95 \%\end{array}$} \\
\hline & $\mathbf{n}$ & $\%$ & $\mathbf{n}$ & $\%$ & \\
\hline \multicolumn{6}{|l|}{ Síndrome nefrítico } \\
\hline $\begin{array}{l}\text { Sí } \\
\text { No }\end{array}$ & $\begin{array}{l}16 \\
20\end{array}$ & $\begin{array}{l}44.4 \\
55.6\end{array}$ & $\begin{array}{l}16 \\
75\end{array}$ & $\begin{array}{l}17.6 \\
82.5\end{array}$ & $\begin{array}{l}<0.01,3.75 \\
(1.6-8.779)\end{array}$ \\
\hline \multicolumn{6}{|l|}{ Afecciones renales } \\
\hline $\begin{array}{l}\text { Sí } \\
\text { No }\end{array}$ & $\begin{array}{l}16 \\
20\end{array}$ & $\begin{array}{l}44.4 \\
55.6\end{array}$ & $\begin{array}{l}31 \\
60\end{array}$ & $\begin{array}{l}34.1 \\
65.9\end{array}$ & $\begin{array}{l}0.187,1.54 \\
(0.70-3.40)\end{array}$ \\
\hline \multicolumn{6}{|c|}{ Alteraciones leucocitarias } \\
\hline $\begin{array}{l}\text { Sí } \\
\text { No }\end{array}$ & $\begin{array}{l}16 \\
20\end{array}$ & $\begin{array}{l}44.4 \\
55.6\end{array}$ & $\begin{array}{c}7 \\
84\end{array}$ & $\begin{array}{c}7.7 \\
92.3\end{array}$ & $\begin{array}{c}<0.01,9.6 \\
(3.48- \\
26.44)\end{array}$ \\
\hline \multicolumn{6}{|l|}{ Púrpura } \\
\hline $\begin{array}{l}\text { Sí } \\
\text { No }\end{array}$ & $\begin{array}{c}1 \\
35\end{array}$ & $\begin{array}{c}2.8 \\
97.2\end{array}$ & $\begin{array}{c}3 \\
88\end{array}$ & $\begin{array}{c}3.3 \\
96.7\end{array}$ & $\begin{array}{c}0.686 \\
0.838 \\
(0.08-8.33)\end{array}$ \\
\hline \multicolumn{6}{|l|}{ Anemia } \\
\hline $\begin{array}{l}\text { Sí } \\
\text { No }\end{array}$ & $\begin{array}{l}11 \\
25\end{array}$ & $\begin{array}{l}30.6 \\
69.4\end{array}$ & $\begin{array}{l}14 \\
77\end{array}$ & $\begin{array}{l}15.4 \\
84.6\end{array}$ & $\begin{array}{c}0.048,2.42 \\
(0.97-6.00)\end{array}$ \\
\hline \multicolumn{6}{|l|}{ Derrame pleural } \\
\hline $\begin{array}{l}\text { Sí } \\
\text { No }\end{array}$ & $\begin{array}{l}13 \\
23\end{array}$ & $\begin{array}{l}36.1 \\
63.9\end{array}$ & $\begin{array}{l}12 \\
79\end{array}$ & $\begin{array}{l}13.2 \\
86.9\end{array}$ & $\begin{array}{l}<0.01,3.72 \\
(1.49-9.26)\end{array}$ \\
\hline \multicolumn{6}{|l|}{ Vasculitis } \\
\hline $\begin{array}{l}\text { Sí } \\
\text { No }\end{array}$ & $\begin{array}{l}19 \\
17\end{array}$ & $\begin{array}{l}52.8 \\
47.2\end{array}$ & $\begin{array}{l}12 \\
79\end{array}$ & $\begin{array}{l}13.2 \\
86.8\end{array}$ & $\begin{array}{c}<0.01,7.35 \\
\quad(3.01- \\
17.96)\end{array}$ \\
\hline
\end{tabular}

Cuadro 3. Principales manifestaciones clínicas del lupus eritematoso sistémico asociadas con mortalidad según otros estudios, acorde al grupo etario de pacientes con este diagnóstico atendidos en dos hospitales de Sonora (2016)* Continuación

\begin{tabular}{|c|c|c|c|c|c|}
\hline \multirow{2}{*}{ Variable estudiada } & \multicolumn{2}{|c|}{$\begin{array}{c}\text { Grupo A } \\
(n=36)\end{array}$} & \multicolumn{2}{|c|}{$\begin{array}{c}\text { Grupo B } \\
(n=91)\end{array}$} & \multirow[b]{2}{*}{$\begin{array}{c}p^{* *} \\
\text { OR IC } 95 \%\end{array}$} \\
\hline & $n$ & $\%$ & $n$ & $\%$ & \\
\hline \multicolumn{6}{|l|}{ Sepsis } \\
\hline $\begin{array}{l}\text { Sí } \\
\text { No }\end{array}$ & $\begin{array}{c}6 \\
30\end{array}$ & $\begin{array}{l}16.7 \\
83.3\end{array}$ & $\begin{array}{c}4 \\
87\end{array}$ & $\begin{array}{c}4.4 \\
95.6\end{array}$ & $\begin{array}{c}0.03,4.35 \\
(1.14- \\
16.47)\end{array}$ \\
\hline \multicolumn{6}{|l|}{ Neumonía } \\
\hline $\begin{array}{l}\text { Sí } \\
\text { No }\end{array}$ & $\begin{array}{c}6 \\
30\end{array}$ & $\begin{array}{l}16.7 \\
83.3\end{array}$ & $\begin{array}{l}81 \\
10\end{array}$ & $\begin{array}{l}89 \\
11\end{array}$ & $\begin{array}{l}0.277,1.62 \\
(0.54-4.84)\end{array}$ \\
\hline \multicolumn{6}{|l|}{ Infecciones } \\
\hline $\begin{array}{l}\text { Sí } \\
\text { No }\end{array}$ & $\begin{array}{l}21 \\
15\end{array}$ & $\begin{array}{l}58.3 \\
41.7\end{array}$ & $\begin{array}{l}26 \\
65\end{array}$ & $\begin{array}{l}28.6 \\
71.4\end{array}$ & $\begin{array}{l}<0.01,3.5 \\
(1.56-7.81)\end{array}$ \\
\hline \multicolumn{6}{|c|}{ Afecciones neuropsiquiátricas } \\
\hline $\begin{array}{l}\text { Sí } \\
\text { No }\end{array}$ & $\begin{array}{c}7 \\
29\end{array}$ & $\begin{array}{l}19.4 \\
80.6\end{array}$ & $\begin{array}{l}26 \\
65\end{array}$ & $\begin{array}{l}28.6 \\
71.4\end{array}$ & $\begin{array}{c}0.204,0.6 \\
(0.23-1.54)\end{array}$ \\
\hline \multicolumn{6}{|c|}{ Afecciones cardiovasculares } \\
\hline $\begin{array}{l}\text { Sí } \\
\text { No }\end{array}$ & $\begin{array}{l}19 \\
17\end{array}$ & $\begin{array}{l}52.8 \\
47.2\end{array}$ & $\begin{array}{l}23 \\
68\end{array}$ & $\begin{array}{l}25.3 \\
74.7\end{array}$ & $\begin{array}{l}<0.01,3.3 \\
(1.47-7.4)\end{array}$ \\
\hline \multicolumn{6}{|c|}{ Afecciones cardiovasculares (sin vasculitis) } \\
\hline $\begin{array}{l}\text { Sí } \\
\text { No }\end{array}$ & $\begin{array}{l}3 \\
33\end{array}$ & $\begin{array}{l}8.3 \\
91.7\end{array}$ & $\begin{array}{l}14 \\
77\end{array}$ & $\begin{array}{l}15.4 \\
84.6\end{array}$ & $\begin{array}{l}0.228,0.5 \\
(0.13-1.85)\end{array}$ \\
\hline
\end{tabular}

*Hospital Infantil del Estado de Sonora (HIES), Hospital General del Estado de Sonora (HGES).

**El valor de $p$ se obtuvo con la prueba de ji cuadrada, excepto en los casos con celdas con valores menores a 5, donde se empleó la prueba exacta de Fisher.

\section{Tratamientos y farmacoterapia}

Se encontraron relaciones significativas entre el grupo etario y el uso de algunos inmunosupresores como tratamiento para el LES. Los valores encontrados para el uso de fármacos en los niños fue como se detalla a continuación: parahidroxicloroquina $(p<0.01)$, glucocorticoides $(p=0.14)$ y micofenolato $(p=0.28)$ azatioprina o ciclofosmfamida dependiendo de la presencia de afección renal. Por su parte, en los adultos los tratamientos fueron con base de 
azatioprina $(p=0.02)$, metotrexato $(p<0.01)$ y ciclofosfamida $(p=0.33)$ (Cuadro 4).

Los niños recibían entre tres y cuatro inmunosupresores en comparación con dos de los adultos $(p<0.01)$. Los niños con LES tienen ocho veces más riesgo de presentar el síndrome de polifarmacia que los adultos con la misma enfermedad $(\mathrm{OR}=8.5$; IC 95\%: 1.7-42.8) (Cuadro 5).

\section{Mortalidad}

Las causas de mortalidad en el LES en 19 adultos del hospital general, considerando a 243 pacientes, fueron las siguientes: 10 por neumonías, 9 por enfermedad renal terminal; de éstas,

Cuadro 4. Fármacos empleados para tratar lupus eritematoso sistémico según el grupo etario en pacientes atendidos en dos hospitales* de Sonora (2016)

\begin{tabular}{|c|c|c|c|c|c|}
\hline \multirow{2}{*}{ Variable estudiada } & \multicolumn{2}{|c|}{$\begin{array}{c}\text { Grupo A } \\
(n=36)\end{array}$} & \multicolumn{2}{|c|}{$\begin{array}{c}\text { Grupo B } \\
(n=91)\end{array}$} & \multirow[b]{2}{*}{$\overline{p^{* *}}$} \\
\hline & $\mathbf{n}$ & $\%$ & $\mathbf{n}$ & $\%$ & \\
\hline \multicolumn{6}{|l|}{ Azatriopina } \\
\hline $\begin{array}{l}\text { Sí } \\
\text { No }\end{array}$ & $\begin{array}{c}8 \\
28\end{array}$ & $\begin{array}{l}22.2 \\
77.8\end{array}$ & $\begin{array}{l}39 \\
52\end{array}$ & $\begin{array}{l}42.9 \\
57.1\end{array}$ & 0.021 \\
\hline \multicolumn{6}{|l|}{ Hidroxicloroquina } \\
\hline $\begin{array}{l}\text { Sí } \\
\text { No }\end{array}$ & $\begin{array}{c}27 \\
9\end{array}$ & $\begin{array}{l}75 \\
25\end{array}$ & $\begin{array}{l}37 \\
54\end{array}$ & $\begin{array}{l}40.7 \\
59.3\end{array}$ & $<0.01$ \\
\hline \multicolumn{6}{|l|}{ Glucocorticoides } \\
\hline $\begin{array}{l}\text { Sí } \\
\text { No }\end{array}$ & $\begin{array}{c}33 \\
3\end{array}$ & $\begin{array}{c}91.7 \\
8.3\end{array}$ & $\begin{array}{l}16 \\
75\end{array}$ & $\begin{array}{l}17.6 \\
82.4\end{array}$ & 0.148 \\
\hline \multicolumn{6}{|l|}{ Metotrexato } \\
\hline $\begin{array}{l}\text { Sí } \\
\text { No }\end{array}$ & $\begin{array}{c}1 \\
35\end{array}$ & $\begin{array}{c}2.8 \\
97.2\end{array}$ & $\begin{array}{l}19 \\
72\end{array}$ & $\begin{array}{l}20.9 \\
79.1\end{array}$ & $<0.01$ \\
\hline \multicolumn{6}{|l|}{ Ciclofosfamida } \\
\hline $\begin{array}{l}\text { Sí } \\
\text { No }\end{array}$ & $\begin{array}{c}3 \\
33\end{array}$ & $\begin{array}{c}8.3 \\
91.7\end{array}$ & $\begin{array}{l}12 \\
79\end{array}$ & $\begin{array}{l}13.2 \\
86.8\end{array}$ & 0.334 \\
\hline \multicolumn{6}{|l|}{ Micofenolato } \\
\hline $\begin{array}{l}\text { Sí } \\
\text { No }\end{array}$ & $\begin{array}{c}3 \\
35\end{array}$ & $\begin{array}{c}7.8 \\
92.2\end{array}$ & $\begin{array}{c}0 \\
91\end{array}$ & $\begin{array}{c}0 \\
100\end{array}$ & 0.283 \\
\hline
\end{tabular}

*Hospital Infantil del Estado de Sonora (HIES), Hospital General del Estado de Sonora (HGES).

*El valor de $p$ se obtuvo con la prueba de ji cuadrada, excepto en los casos con celdas con valores menores a 5, donde se empleó la prueba exacta de Fisher.
Cuadro 5. Cantidad de inmunosupresores empleados de forma concomitante (y síndrome de polifarmacia) en pacientes con diagnóstico de lupus eritematoso sistémico atendidos en dos hospitales* de Sonora (2016)

\begin{tabular}{|l|c|c|c|c|c|}
\hline \multirow{2}{*}{ Variable estudiada } & \multicolumn{2}{|c|}{$\begin{array}{c}\text { Grupo A } \\
(\mathbf{n = 3 6 )}\end{array}$} & \multicolumn{2}{c|}{$\begin{array}{c}\text { Grupo B } \\
(\mathbf{n = 9 1 )}\end{array}$} & \\
\cline { 2 - 6 } & $\mathbf{n}$ & $\%$ & $\mathbf{n}$ & $\%$ & $\overline{\boldsymbol{p}^{* *}}$ \\
\hline Cantidad de inmunodepresores & & & & \\
\hline Ningún fármaco & 0 & 0 & 9 & 9.9 & $<0.01$ \\
\hline Monoterapia & 2 & 20 & 16 & 17.6 & \\
\hline Dos fármacos & 0 & 0 & 37 & 40.7 & \\
\hline Tres fármacos & 4 & 40 & 24 & 26.4 & \\
\hline Cuatro fármacos & 3 & 30 & 5 & 5.5 & \\
\hline Cinco fármacos & 1 & 10 & 0 & 0 & \\
\hline Polifarmacia & & & & & \\
\hline Sí & 8 & 80 & 29 & 68.1 & $<0.01$ \\
\hline No & 2 & 20 & 62 & 31.9 & \\
\hline
\end{tabular}

*Hospital Infantil del Estado de Sonora (HIES), Hospital General del Estado de Sonora (HGES).

**Se tomó el valor de $p$ hacia ambos lados de la curva en la variable cantidad de inmunosupresores. El valor de $p$ se obtuvo realizando la ji cuadrada, y en los casos donde hay celdas con valores menores a 5, se empleó la prueba exacta de Fisher.

5 correspondieron a los 91 pacientes incluidos en este estudio; los otros 5 por causas diversas tales como: meningitis por coccidioidesimmitis, gastroenteritis infecciosa, sangrado de tubo digestivo, insuficiencia hepática aguda y septicemia. En los niños, en cambio, de 9 decesos registrados 7 fueron por neumonía, uno por septicemia y uno por insuficiencia renal.

En resumen, el número de muertes en el período de estudio; en 91 adultos fue de $5.4 \%$ y las ocurridas en el hospital pediátrico en 36 niños, fue en $9(25 \%)$.

\section{DISCUSIÓN}

El porcentaje de los pacientes que manifiestan esta enfermedad en la infancia para grupos hispánicos se reporta en $20 \%{ }^{14,15}$ En esta serie 28\% 
de los pacientes analizados fueron niños, aunque la muestra corresponde a un hospital pediátrico de concentración no necesariamente representa lo que acontece para todo es estado.

Se observó que en el grupo A la relación entre hombre:mujer fue 1:2.6 y en el B fue de 21.7:1, lo cual ha sido reportado previamente en estudios que incluyeron población de origen mexicano. ${ }^{6,15,16}$

Se ha reportado una prevalencia de $77 \%$ para las manifestaciones cutáneas en niños, en esta muestra se presentó en $72 \% .^{17,18} \mathrm{El}$ porcentaje reportado de pacientes con LES que presentan fotosensibilidad coincide con esta serie de $60 \% .^{19}$

A la nefritis lúpica se le cuenta entre las causas de mortalidad en pacientes con LES, se le considera un factor de riesgo importante para desarrollar enfermedad renal crónica. ${ }^{14,20,21}$ La prevalencia de nefritis en niños fue menor a la reportada en Medio Oriente y en Estados Unidos ${ }^{18,22,23}$ pero, aún así, fue significativamente mayor que en los adultos. Los factores de mal pronóstico para niños con nefritis lúpica incluyen: proteinuria persistente, hipertensión arterial al inicio de la enfermedad, falta de remisión completa en el primer año de tratamiento, nivel de C3 bajo, creatinina elevada y origen étnico no caucásico; además de una clasificación histológica grados IV-V. ${ }^{14}$ Por ello es necesario vigilar de cerca estos parámetros en pacientes de edad pediátrica.

Otros estudios han relacionado algunas manifestaciones hematológicas como factores de mal pronóstico, la anemia trombocitopenia ocurre en la edad pediátrica entre 55 y $75 \%$ de los casos; de éstas, la leucopenia ocurre en 25 a $52 \%$, e incluso con un mayor riesgo de muerte. ${ }^{10,24,25}$ Esto lo encontramos en el grupo B en forma más frecuente y con diferencia significativa en las siguientes manifestaciones hematológicas: trombocitopenia (25\% de los niños contra $7.7 \%$ del grupo A), anemia (30.6\% contra $15.4 \%$ ) y alteraciones leucocitarias (44\% contra $7.7 \%$ ).

La trombocitopenia se asocia indirectamente con mayor actividad de la enfermedad $y$, por lo tanto, con tendencia a presentar mayor daño orgánico. ${ }^{26-29}$

La anemia se puede deber a múltiples causas: a la propia actividad lúpica, deficiencia de eritropoyetina por enfermedad renal crónica y pérdida por sangrados del tubo digestivo, entre otras. ${ }^{30}$ La anemia hemolítica se ha asociado con mayor mortalidad y se reporta con mayor frecuencia en niños que en adultos.

La leucopenia también se ha asociado indirectamente con mayor actividad de la enfermedad y con otras manifestaciones como úlceras orales, infecciones y serositis, además de ser un factor predisponente para padecer enfermedades infecciosas que pueden ser mortales. ${ }^{31}$

Otro factor que se ha asociado indirectamente con la mortalidad en pacientes con LES es la serositis. ${ }^{24} \mathrm{El}$ derrame pleural se presenta hasta en la mitad de los pacientes y se debe a la enfermedad misma o a complicaciones como insuficiencia renal, insuficiencia cardíaca o infecciones, las cuales pueden conducir a la muerte $\mathrm{e}^{32,33}$ por lo que se requiere un juicio clínico meticuloso para identificarlas.

La enfermedad cardiovascular es otra de las manifestaciones que se relacionan con la muerte de los pacientes. ${ }^{11,13,34}$ La vasculitis en el LES varía del 11 al 20\%. Las vasculitis viscerales pueden evolucionar a la gravedad y conducir a la muerte al causar lesiones isquémicas en cerebro, corazón o intestinos, por mencionar algunos. ${ }^{35,36} \mathrm{En}$ los casos en que no causan la muerte, la vasculitis, sobre todo a nivel abdominal, Ileva muy frecuentemente a un deterioro en la calidad de vida, al causar en los pacientes dolor crónico 
intenso y de difícil tratamiento. Una detección temprana y un tratamiento oportuno mejoran el pronóstico, por lo que hay que tener presente esta afección en pacientes con la enfermedad y abdomen agudo. ${ }^{37-39}$

Las infecciones constituyen la principal causa de mortalidad en los países en vías de desarrollo; las principales razones que se aducen, para la predisposición de los pacientes a una infección, son el tratamiento inmunosupresor y las alteraciones inmunológica propias del lupus. ${ }^{40-42}$ Los niños hospitalizados con altos títulos de anti-DNA, niveles bajos del complemento y una mayor actividad de la enfermedad son más propensos a sepsis, por lo que es recomendable identificarlos por clínica y laboratorio. ${ }^{43}$

En la medida que son detectadas más complicaciones y manifestaciones de alta actividad de la enfermedad la asociación con lupus neuropsiquiátrico es mayor; ${ }^{44}$ afecta notablemente la calidad de vida de los pacientes y se le correlaciona con mayor riesgo de suicidio. ${ }^{45}$ Se ha evidenciado también que las manifestaciones neuropsiquiatrícas ocurren tanto en niños como en adultos. ${ }^{46}$ En esta muestra no utilizamos escalas de evaluación para el LES del tipo Lupus Erythematous Activity Index (SLEDAI) para medir actividad y establecer relación de casos con afecciones neuropsiquiátricas; por lo tanto, no es posible hacer inferencias al respecto. ${ }^{47}$

De acuerdo con los hallazgos clínicos observamos en los niños mayor actividad de la enfermedad y que en ellos se emplean más fármacos inmunosupresores en comparación con los adultos; esto coincide con otros reportes. . $^{3,48,49}$ Esto puede asociarse con una mayor cantidad de efectos adversos en ese grupo de pacientes; circunstancia que debe ser analizada específicamente y conocer su relación con la mortalidad de $25 \%$ reportada en niños en nuestro medio de trabajo. ${ }^{3,6,48-53}$
Existe poco información en la literatura sobre el síndrome de polifarmacia en niños con lupus; además, la terapia se basa, principalmente, en hallazgos de ensayos clínicos realizados en adultos. ${ }^{48-53}$

\section{CONCLUSIONES}

El lupus eritematoso sistémico es una enfermedad inflamatoria crónica y autoinmune que afecta con mayor frecuencia a adultos, pero con mayor severidad a niños.

En este análisis fue posible identificar que los niños tienen más riesgo de presentar manifestaciones capaces de conducirlos a la muerte: vasculitis, nefritis lúpica, alteraciones leucocitarias y derrame pleural. Por ello, es prudente tener un mayor control dirigido a esas complicaciones monitoreando periódicamente y de forma rigurosa, particularmente las relacionadas a su función renal, es indispensable la búsqueda de proteinuria, conteo de células sanguíneas, niveles del complemento y una búsqueda intencionada de datos clínicos de infección y serositis.

Finalmente, el síndrome de polifarmacia y el uso de inmunosupresores para tratar el LES en niños deben analizarse por separado y ponderarse con cautela, considerando siempre su costobeneficio, sobre todo mientras la terapia infantil esté basada en la evidencia para adultos.

\section{AGRADECIMIENTOS}

Los autores agradecen la asesoría científica y la ayuda técnica de: Dra. Ana Lourdes Mata Pineda, Dr. Carlos Arturo Velázquez Contreras y Dr. Juan Carlos Núñez Enríquez del Departamento de Medicina de la Universidad de Sonora. Agradecemos también la asesoría y apoyo brindado por: Dra. Luz María Suárez Larios, Reumatóloga del Hospital Infantil de Estado de Sonora y Dr. Jorge Isaac Cardoza Amador, Ex Director Médico del Hospital General del Estado. 


\section{REFERENCIAS}

1. Pons-Estel G, Catoggio L, Cardiel M, Bonfa E, Caeiro F, Sato $E$ et al. Lupus in Latin-American patients: lessons from the GLADEL cohort. Lupus. 2015;24(6):536-545.

2. Pelaez-Ballestas I, Sanin L, Moreno-Montoya J, AlvarezNemegyei J, Burgos-Vargas R, Garza-Elizondo $\mathrm{M}$ et al. Epidemiology of the Rheumatic Diseases in Mexico. A Study of 5 Regions Based on the COPCORD Methodology. The Journal of Rheumatology Supplement. 2011;86(0):3-8.

3. Blancas L, Guevara M, Espinosa F. Lupus Eritematoso Sistémico en niños, una revisión de la supervivencia. Alerg. Asma Inmunol. Pediatr. 2009;18(1):6-9.

4. Badillo-Tenorio RE, Rivas-Larrauri FE. Lupus eritematoso sistémico y gammaglobulina intravenosa. Acta Pediatr Mex. 2013;34(6):353-357.

5. Sotelo-Cruz N, Ibarra-Silva R, Monge- López L, Hurtado- Valenzuela JG. Veintiocho años de experiencias en el manejo de niños con lupus eritematoso sistémico: Revisión de 26 casos. Rev Mex Pediatr. 2006;73(2):60-66.

6. Camacho MS, Lirola JM. Lupus eritematoso sistémico. An Pediatr Contin 2007;5(3):123-130.

7. Hernandez B, Tapia N, Villa A, Reyes E, Cardiel M. Risk factors associated with mortality in systemic lupus erythematosus. A case-control study in a tertiary care center in Mexico City. Clinical and Experimental Rheumatology. 2001;19:395-401.

8. Rees F, Doherty M, Grainge M, Lanyon P, Davenport G, Zhang W. Mortality in systemic lupus erythematosus in the United Kingdom 1999-2012. Rheumatology. 2016;55(5):854-60.

9. Wang Z, Wang Y, Zhu R, Tian X, Xu D, Wang Q et al. LongTerm Survival and Death Causes of Systemic Lupus Erythematosus in China. Medicine. 2015;94(17):e794.

10. Lee $\mathrm{Y}$, Choi S, Ji J, Song G. Overall and cause-specific mortality in systemic lupus erythematosus: an updated meta-analysis. Lupus. 2016;25(7):727-734.

11. Kasitanon N, Magder L, Petri M. Predictors of Survival in Systemic Lupus Erythematosus. Medicine. 2006;85(3):14756.

12. Tavangar-Rad F, Ziaee V, Maradinej M, Tahghighi F. Morbidity and Mortality in Iranian Children with Juvenile Systemic Lupus erythematosus. Iran. J Pediatr. 2014;24(4):365-370.

13. Bartels C, Burh K, Goldberg J, Bell C, Visekruna M, Nekkanti $S$ et al. Mortality and Cardiovascular Burden of Systemic Lupus Erythematosus in a US Population-Based Cohort. J. Rheumatol. 2014;41(4):680-87.

14. 14. Bernatsky S, Bolvin J, Joseph L, Manzi S, Ginzler E, Gladman D et al. Mortality in Systemic Lupus Erythematosus. Arthritis \& Rheumatism. 2006;54(8):2550-57.

15. Blancas L, Guevara M, Berrón R, Berrón L, Gutiérrez $P$, Espinosa F. Survival of Mexican patients with paediatriconset systemic lupus erythematosus and abnormal elec- troencephalogram. Allergol Inmmunopathol (Madrid). 2013;41(2):108-13.

16. Velázquez-Cruz R, Jiménez-Morales $S$, Ramirez-Bello J, Aguilar-Delfín I, Salas- Martinez G, Baca-Ruiz V et al. Lupus eritematoso sistémico (LES): genómica de la enfermedad. Gaceta Médica de México (México). 2012;148(4):371-380.

17. Zonana-Nacach $A$, Rodríguez-Guzmán L, Jiménez- Balderas F, Camargo-Coronel A, Escobedo de la Peña J, Fraga A. Factores de riesgos relacionados con lupus eritematoso sistémico en población mexicana. Salud Pública de México (México). 2002;44(3):213-218.

18. Wananukul S, Watana D, Pongprasit P. Cutaneous Manifestations of Childhood Systemic Lupus Erythematosus. Pediatric Dermatology. 1998;15(5):342-346.

19. Malattia C, Martini A. Paediatric-onset systemic lupus erythematosus. Best Practice and Research Clinical Rheumatology. 2013;27(3):351-62.

20. Werth V, Bashir M, Zhang W. Photosensitivity in Rheumatic Diseases. J. Investig. Dermmatol. 2004;9(1):57-63.

21. Pokroy-Shapira E, Gelernter I, Molad Y. Evolution of chronic kidney disease in patients with systemic lupus erythematosus over a long-period follow-up: a single-center inception cohort study. Clin. Rheumatol. 2014:33(5):644-57.

22. Bogdanovic R, Nikolić V, Pašić S, Dimitrijević J, LipkovskaMarković J, Erić-Marinković J et al. Lupus nephritis in childhood: a review of 53 patients followed at a single center. Pediatr. Nephrol. 2004;19(1):33-44.

23. Elmougy A, Sarhan A, Hammad A, El-Refaey A, Zedan M, Eid $R$ et al. Lupus nephritis in Egyptian children: a 16-year experience. J. Nephrol. 2014;28(5):557-62.

24. Bennett M, Brunner H. Biomarkers and Updates on Pediatrics Lupus Nephritis. Rheum. Dis. Clin. 2013;39(4):833-53.

25. Yeap S, Chow S, Manivassagar M, Veerapen K, Wang F. Mortality patterns in Malaysian systemic lupus erythematosus patients. Med. J. Malaysia. 2001;56(3):308-12.

26. Abu-Shakra M, Urowitz M, Gladman D, Gough J. Mortality studies in systemic lupus erythematosus. Results from a single center. II. Predictor variables for mortality. J. Rheumatol. 1995;22(7):1259-64.

27. Zhao H, Li S, Yang R. Thrombocytopenia in patients with systemic lupus erythematosus: significant in the clinical implication and prognosis. Platelets. 2010;21(5):380-385.

28. Fernandez M, Alarcón G, Apte M, Andrade R, Vilá L, Reveille J. Systemic lupus erythematosus in a multiethnic US cohort: $X$ LIII. The significance of thrombocytopenia as a prognostic factor. Arthritis. Rheum. 2007;56(2):614-621.

29. Patel N, Mody M. Acute presentation of thrombocytopaenia in systemic lupus erythematosus is associated with a high mortality in South Africa. Lupus. 2014;23(2):204-12.

30. Ktona E, Barbullushi M, Backa T, Idrizi A, Shpta V, Roshi E. Evaluation of Thrombocytopenia in Systemic Lupus Erythematosus and Correlation with Different Organs Damages. Mater. Sociomed. 2014:26(2):122-24. 
31. Lam S, Quah T. Anemia in systemic lupus erythematosus. J. Singapore Paediatr. Soc. 1990;32(3-4):132-36.

32. Aemer A, Al A, Khalil N, Alarfaj H.Haematological abnormalities in systemic lupus erythematosus. Acta Reumatol Port. 2014;39(3):236-41.

33. Fekih L, Boussoffara L, Chaouachi S, Fenniche S, Abdelghaffar $\mathrm{H}$, Akrout I et al. Thoracic manifestations of systemic lupus erythematosus. La tunisie Medicale. 2011;89(3):269-73.

34. Torre $\mathrm{O}$, Harari S. Pleural and Pulmonary Inovolvement in Systemic Lupus Erithematosus. La Presse Medical. 2011:40(1):19-29.

35. Gustafsson J, Simard J, Gunnarsson I, Elvin K, Lundberg I, Hansson L et al. Risk factors for cardiovascular mortality in patients with systemic lupus erythematosus, a prospective cohort study. Arthritis. Res. Ther. 2012;14(2):e46.

36. Ramos M, Nardi N, Lagrutta M, Brito P, Bové A, Delgado G et al.Vasculitis in Systemic Lupus Erythematosus Prevalence and Clinical Characteristics in 670 Patients. Medicine. 2006;85(2):95-104.

37. Drenkard C. Thrombocytopenia as a prognostic factor in Hispanic patients with systemic lupus erythematosus: comment on the article. Emory University Atlanta, GA 2007.

38. Radic M, Martinovic D, Radic J. Vascular manifestations of systemic lupus erythematosus. Neth. J. Med. 2013;71(1):10-6.

39. Toubi E, Kessel A, Bamberger E, Golan T. Systemic Lupus Erythematosus Vasculitis: A Current Therapeutic Overview. Curr. Treat Options Cardiovasc. Med. 2004;6(2):87-7.

40. Lampros F, Baszis K, French A, Cooper M, White A. Mesenteric vasculitis in children with systemic lupus erythematosus. Clinical Rheumatology. 2015;35(3):785-93.

41. Mohamed D, Habeeb R, Hosny S, Ebrahim S. Incidence and Risk of Infection in Egyptian Patients with Systemic Lupus Erythematosus. Clinical Medicine Insights: Arthritis and Musculoskeletal Disorders. 2014;7:41-8.

42. Marcos M, Fernández $C$, Soriano A, Marco F, Martínez A, Almela $M$, et al. Epidemiology and clinical outcomes of bloodstream infections among lupus patients.Lupus. 2011;20(9):965-971.

43. Chen $\mathrm{M}$, Tseng $\mathrm{H}$, Huang $\mathrm{Y}$, Hsu $\mathrm{W}$, Yeh $\mathrm{K}$, Wu T et al. Longterm outcome and short-term survival of patients with systemic lupus erythematosus after bacteremia episodes: 6-yr follow-up. Rheumatology. 2008;47(9):1352-57.

44. Chen $Y$, Yang $Y$, Lin Y, Chiang B. Risk of infection in hospitalized children with systemic lupus erythematosus: a 10-year follow-up. Clin. Rheumatol. 2004;23(3):235-38.

45. Abdul-Sattar A, Goda T, Negm M. Neuropsychiatric manifestations in a consecutive cohort of systemic lupus erythematosus; a single center study. International Journal of Rheumatic Diseases. 2013;16(6):715-23.

46. Hajduk A, Nowicka K, Smoleńska Ż, Czuszyńska Z, Zdrojewski Z. Prevalence and correlates of suicidal thoughts in patients with neuropsychiatric lupus. LUPUS. 2016;25(2):182-92.

47. Miniño M. Indice de actividad Lupica y tratamiento del Lupus eritematoso en dermatología .Dermatol Rev Mex. 2008;52(1):20-28.

48. Kohut S, Williams T, Jayanthikumar J, Landolt-Marticorena C, Lefebvre A, Silverman E et al. Depressive symptoms are prevalent in childhood-onset systemic lupus erythematosus (cSLE). LUPUS. 2013;22(7):712-21.

49. León T, Henríquez C, Calderón J, Massardo L. Actualización en lupus neuro-psiquiátrico con énfasis en déficit cognitivo. Rev. Med. Chile. 2012;140(10):1333-41

50. Mina R, Brunner H. Update on differences between childhood- onset and adult-onset systemic lupus erythematosus. Arthritis Res. Ther. 2013;15(4):e218.

51. Arici Z, Batu E, Ozen S. Reviewing the Recommendations for Lupus in Children. Curr. Rheumatol Rep. 2015;17(39):e489.

52. Pavón-Sánchez JM, Sánchez-Sánchez LM. Evolución de pacientes pediátricos con lupus eritematoso sistémico tratados con rituximab. Gac Med Mex 2013;149(5):492-496.

53. Teh C, Ling G. Causes and predictors of mortality in hospitalized lupus patient in Sarawak General Hospital, Malaysia. Lupus. 2013;22(1):106-11. 